Article

\title{
The Immobilization of Oxindole Derivatives Using New Designed Functionalized C60 Nanomolecules
}

\author{
Przemysław Czeleń *(i) and Beata Szefler(i) \\ Department of Physical Chemistry, Faculty of Pharmacy, Collegium Medicum, Nicolaus Copernicus University, \\ Kurpinskiego 5, 85-096 Bydgoszcz, Poland; beatas@cm.umk.pl \\ * Correspondence: przemekcz@cm.umk.pl
}

Received: 23 March 2020; Accepted: 8 April 2020; Published: 17 April 2020

\begin{abstract}
Indirubin and its analogs such as oxindole derivatives are well known as competitive inhibitors of cyclin-dependent kinase 2 (CDK2) and play an important role in the creation of therapies in many cancer diseases. Recent research, in order to increase efficiency, is aimed at creating targeted therapy, which is often based on the immobilization of drugs on the surface of nanocarriers. In this work, two oxindole derivatives were used to test the binding capabilities of newly in silico designed C60 fullerene derivatives. Seventy functionalized nanostructures were created by the addition of amino acid substituents to the single phenyl ring attached to the fullerene surface. Realized calculations, based on flexible docking methods, allowed for obtaining energetic characteristics and structural aspects of complexes created by nanomolecules with considered ligands. Analysis of obtained complexes shows that symmetric substitution to position R3 and R5 allows obtaining fullerene derivatives exhibiting the highest binding capabilities, while the lowest ones are the effect of asymmetric substitution (R2; R4). Obtained values clearly allowed to select a group of substituents and substitution sites that provide the most stable complexes which can be used to create new nanocarriers for the group of drugs under consideration.
\end{abstract}

Keywords: CDK2; oxindole derivatives; docking; C60 fullerene; targeted therapy; drug immobilization

\section{Introduction}

One of the significant challenges in current research, placed on the border between medicine and pharmacy is not only the discovery of drugs, but also the creation of appropriate therapies, which allow for maximum utilization of the pharmacological potential of substances by increasing the selectivity and eliminating the undesirable effects of their action. The development of targeted therapy is frequently related to immobilization of drug molecules by molecular carriers. Such a role can be fulfilled by different classes of molecules like albumin [1], chitosan [2,3], gelatin [4], gold complexes [5,6], hydrogels [7], magnetic iron oxides [8] polymers [9-11], cube rhombellanes [12-14] and fullerenes and their derivatives [15-18]. The implementation of such types of methods ensures the direct delivery of drugs and their release to the appropriate biological target. In addition, additional effects can be observed such as increasing drug bioavailability, elongation of pharmacological action $[19,20]$ and decreasing of toxicity $[16,21]$. Recently, one of the most dynamically developing groups of nanoparticles, applicable not only in medicine and pharmacy, are fullerenes. One of the most commonly used molecules in this group is fullerene C60 [15-18,22]. The characteristic chemical structure of this molecule increases the binding capacity toward biologically active molecules containing aromatic systems. This nanoparticle exhibits biological activity toward the cells of living organisms [17], and good permeation through the cell membrane [23-26], and additionally, small concentrations of this compound are nontoxic for living organisms. This nanoparticle provides a good platform for developing new derivatives $[27,28]$ obtained by functionalization of the native system with the use of active groups 
which can significantly increase binding capabilities of such nanocarriers. The type and quantity of active groups used in such processes should be fitted to the chemical and structural properties of immobilized molecules, which enables the maximal efficiency of the obtained nanomolecules. The oxindole derivatives are known as inhibitors of enzymes included in the group of cyclin-dependent kinases [29-31]. The biological activity of these compounds plays an important role in the creation of therapies used in many cancer diseases. Examples of such compounds are indol_4_9 and indol_20_10 which exhibit activity toward the cyclin-dependent kinase 2 (CDK2) enzyme [32]. The chemical structure of both considered molecules (Figure 1) consists of an oxindole core and two side chains, each of them includes a cyclic system in its structure. Hydrogen bond donors and acceptors are also present. Previous research has shown that an important factor that can contribute to improved binding of such molecules by fullerene $\mathrm{C} 60$ derivatives is the addition of aromatic rings to the fullerene surface. Additional groups able to participate in hydrogen bond creation are also desirable [14,32].

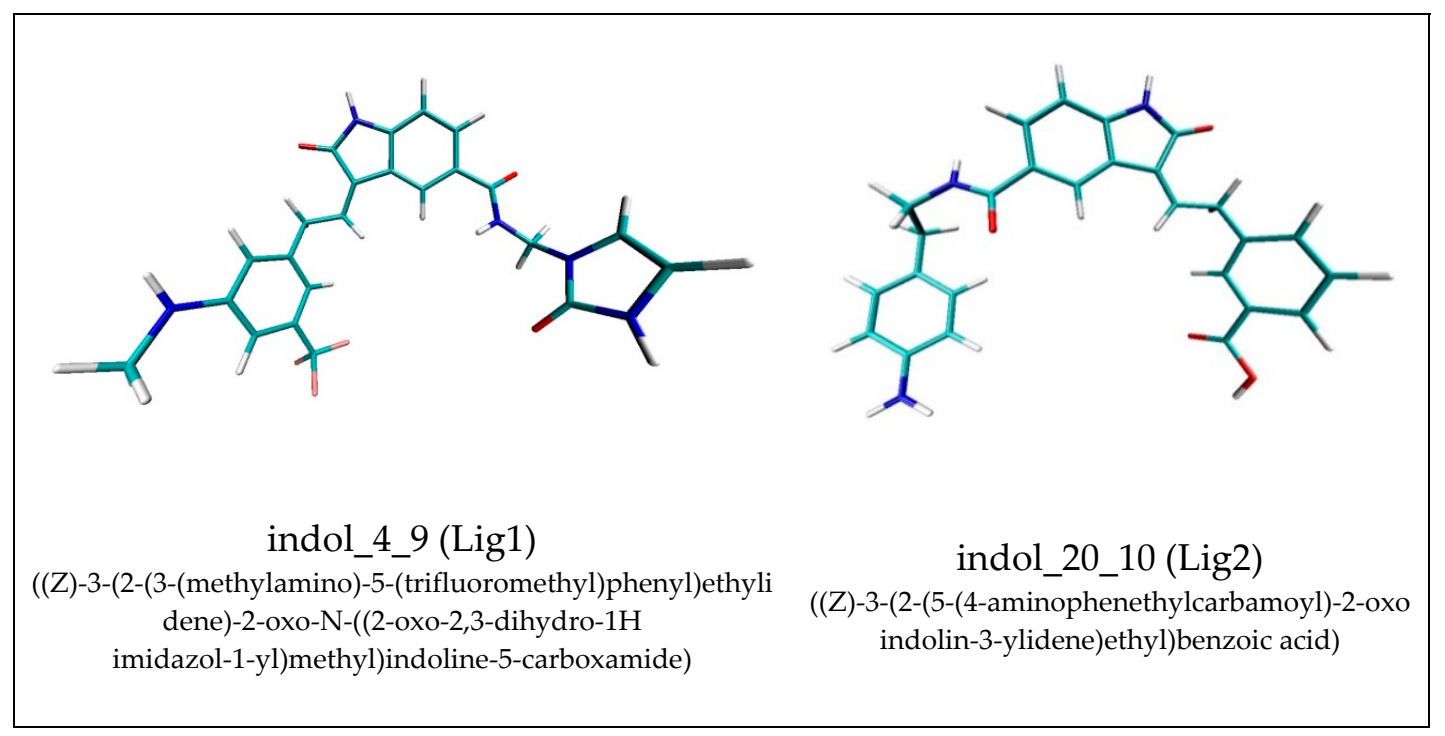

Figure 1. Graphic representations of the ligand molecules used during immobilization. The atoms of particular chemical elements are indicated by the following colors. Carbon: cyan; nitrogen: blue; oxygen: red; hydrogen: white; fluorine: pink.

The use of a single additional aromatic system on the fullerene surface changes its binding capabilities, however further modifications of such systems use the potential of interactions of a larger number of active ligand groups. Amino acids are an important group of compounds that are characterized by significant chemical diversity and, simultaneously, are completely neutral to organisms. Such types of substituents were used in many studies related to the modification of fullerenes [27,33]. The use of a selected group of these compounds can provide substituents containing both hydrogen bond donors and acceptors, as well as additional cyclic systems, particularly important in the immobilization of the ligands proposed in this paper. The addition of such substituents to the fullerene surface may cause an increase of binding capability of the obtained nanomolecules. In such cases, the mobility of the substituent and the possibility of the active interaction with the ligand molecule will be limited. A more convenient target of addition than the fullerene surface appears to be the aromatic ring placed on it. The possibility of choosing a place of substitution, and eventually multiple substitution by residues with flexible molecular chains, gives a chance to obtain a set of functionalized fullerenes exhibiting a high degree of matching to a particular drug molecule. Newly developed nanocarriers based on amino acids may show significant potential in creating targeted therapies based on the discussed group of drugs. 


\section{Methods}

The ligand molecules used during docking stage, namely indol_4_9 (Lig1) ((Z)-3-(2-(3-(methylamino)-5-(trifluoromethyl)phenyl)ethylidene)-2-oxo-N-((2-oxo-2,3-dihydro-1H imidazol-1-yl)methyl)indoline-5-carboxamide) and indol_20_10 (Lig2) ((Z)-3-(2-(5-(4-aminophenethylcarbamoyl)-2-oxoindolin-3-ylidene)ethyl)benzoic acid), were identified as potential competitive inhibitors of CDK2 protein. The C60 structure was downloaded from the Brookhaven Protein Database PDB [34], while C60 functionalized derivatives were created using the Discovery Studio 4.1 package by modifications of CID_71619159 molecule downloaded from the PubChem database [35]. The functionalization of C60 fullerene was realized with the use of the chosen amino acids, which were bound by a peptide bond to a benzene ring attached to the surface of the nanoparticle. According to the diagram in Figure 2, three substituted derivatives were created, resulting in a total of 70 functionalized C60 fullerene derivatives (FF).

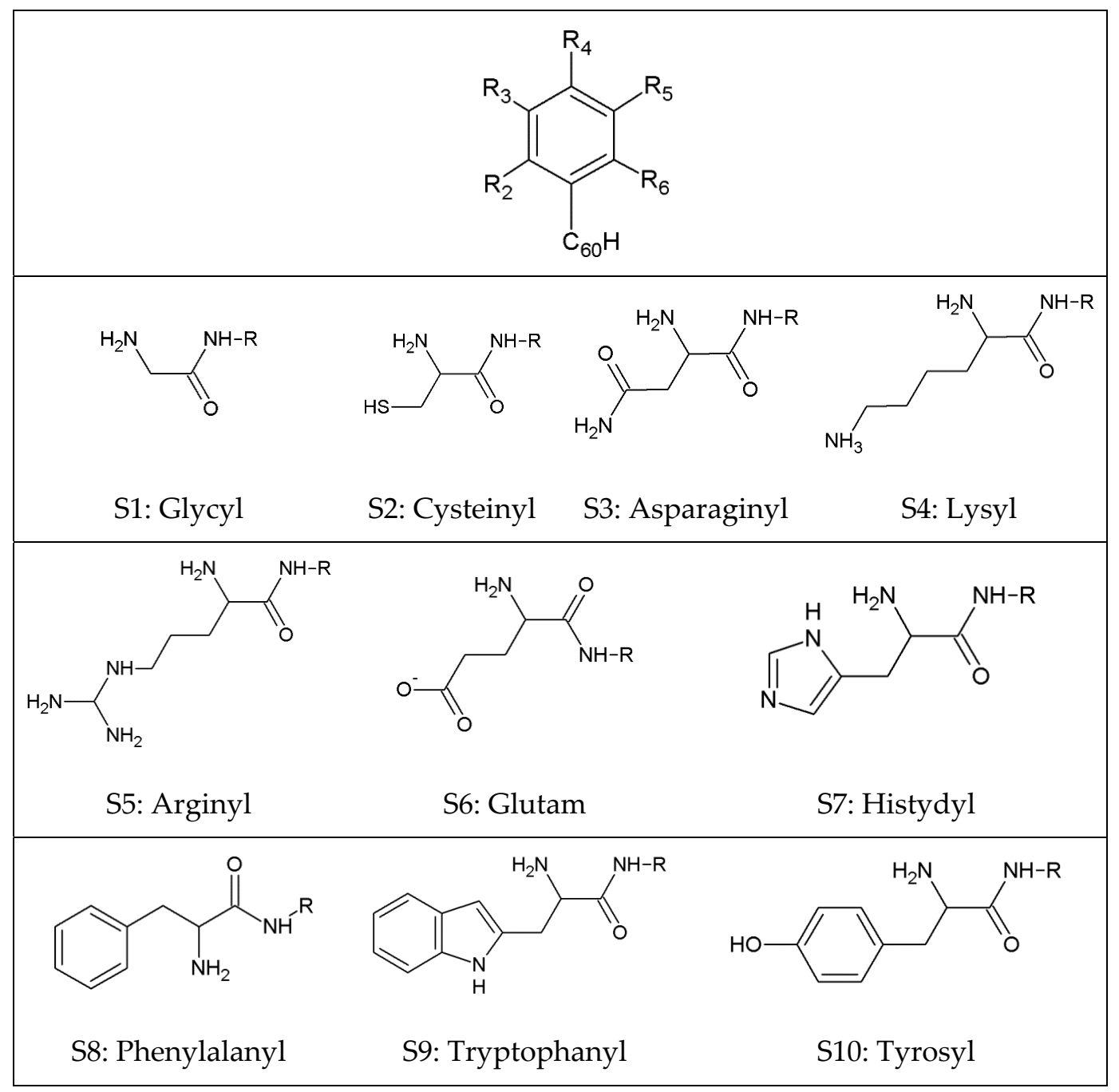

Figure 2. The graphic representation of created functionalized C60 fullerene derivatives (FF). The descriptions Rx define the place of addition of the substituent. The native molecule C60_phen has hydrogen atoms in all substitution areas. The abbreviations from S1 to S10 define particular substituents.

The united-atom scoring function implemented in the AutoDockVina program [36] was used during the docking stage. All structures used during simulations, namely both ligands and all nanoparticles, contained only polar hydrogen atoms. In the case of all considered nanosystems, flexible docking calculations were realized enabling the possibility of the rotation of amino acid substituents 
relative to the benzene ring. For all considered systems, the grid box dimensions were established equal to $32 \times 32 \times 32 \AA$. All initial procedures related to the preparation of the ligands and nanomolecules were realized with the use of the AutoDock Tools package [37]. The exhaustiveness parameter equal to 20 was used for all calculations during the docking stage, since such a value ensures an appropriate reproducibility of the results and a reasonable time of calculation. The analysis of structural properties and visualization of complexes obtained during simulation were realized with the use of the VMD package [38]. Numerous comparative studies clearly show that among the available docking tools, AutoDock Vina exhibits the highest structural reproducibility, and in the case of affinity calculations, it is one of the most accurate [39]. The experimentally estimated average error of Gibbs energy calculation of the method implemented in AutoDock Vina is $2.75 \mathrm{kcal} / \mathrm{mol}$, a value obtained based on measurements for 116 complexes of ligands with highly diversified chemical structures (quantity of ligand rotatable bonds ranging from 0 to 35) [36]. Both the elements of the systems analyzed in this work, namely ligands and proposed nanomolecules, exhibit significant chemical consistency. Therefore, the obtained results should reliably describe the differences between the compared systems. In the case of all considered complexes, in order to check the reproducibility of calculations, multiple docking runs were realized allowing to gather 27 conformers of each analyzed complex, and the energetic diversity of obtained populations was described by RMSD values placed in the range from 0.06 to $0.24 \mathrm{kcal} / \mathrm{mol}$. Structures described by the highest affinity values for each population were selected for structural and energetic analysis.

\section{Results and Discussion}

The chemical structure of both considered ligand molecules consists of an oxindole core and two side chains containing aromatics systems. In addition, the presence of hydrogen bond donors and acceptors in each molecule is of importance. Such chemical properties of considered molecules determine the type of interactions that stabilize their complexes with nanoparticles. In the case of the most often used molecule, namely fullerene C60, only stacking interactions are possible between aromatic systems of ligands and the surface of the fullerene. The functionalization of classic fullerene molecules allows for increasing binding capacity of the considered molecules by the addition of active groups providing a wider range of interaction types with ligand molecules. The attachment of a single benzene ring to the surface of the $\mathrm{C} 60$ molecule has significantly increased the binding affinity of the ligands toward the nanoparticle (Lig1 6.4 and Lig2 14.9 times increase of binding constant). Such a nanostructure was a starting point in the creation of new derivatives obtained by the addition of amino acid substituents to the benzene ring. The binding affinities and binding constants characterizing complexes obtained during docking stages are presented in Tables 1 and 2. The presented values highlight several important dependencies related to the ligand molecule, as well as the type and localization of the used substituents in the aromatics system. In the case of both ligands, there are observed complexes in which the functionalization of nanostructures only slightly influenced the binding constant, as well as systems with a significant increase of this value. The use of substituents with quite a short carbon chain like glycine, cysteine or asparagine monomers does not significantly affect the affinity of these structures toward the ligands. What is important, however, is that a change in the number or location of these substituents in the aromatic system does not significantly affect the binding capacity, which is confirmed only by slight changes in the binding constant. The highest discrepancies are observed for the asparaginyl substituent; however, in this case, the differences between the compared nanostructures do not exceed a twofold increase of the binding constant $(\mathrm{Kx} / \mathrm{Kxmin}=1.96$; Lig2). Significant effects of benzene ring substitution are observed for the next group of residues namely lysine, arginine and glutamine. values observed characterizing the binding affinity and constant, which describes the complexes created by a particular FF. The smallest binding capabilities are exhibited by monosubstituted derivatives. Taking into account the position of the substituent, the binding capacities for most molecules increase in the following order: $\mathrm{R} 4<\mathrm{R} 2<\mathrm{R} 3$. The FF with two and three substituents exhibits different binding properties relative to the considered 
ligands. In the case of the Lig1 molecule, the smallest binding activity is observed in the case of derivatives with substituents placed in positions R2 and R4, and an increase of affinity is observed in the case of substitution in positions R2 and R6.

Table 1. The values of binding affinity $\Delta \mathrm{G}(\mathrm{kcal} / \mathrm{mol})$ of the Lig1 molecule toward the proposed FF; $\mathrm{K}\left(10^{6}\right)$ represents values of the binding constant; $K x / K C 60 p$ represent normalized factors describing an increase of the binding constant relative to the reference structure (C60_phen; $\Delta \mathrm{G}=-8.0 \mathrm{kcal} / \mathrm{mol}$; $\left.\mathrm{K}=0.731 \times 10^{6}\right) ; \mathrm{K} x /$ Kxmin represent normalized factors describing an increase of the binding constant relative to the smallest values obtained for the functionalized fullerene with a particular substituent.

\begin{tabular}{|c|c|c|c|c|c|c|c|c|}
\hline \multirow{2}{*}{\multicolumn{2}{|c|}{$\begin{array}{l}\text { Quantity and Localization of } \\
\text { Substituents }\end{array}$}} & \multicolumn{7}{|c|}{ FF } \\
\hline & & $R 2 ; R 4 ; R 6$ & $R 2 ; R 4$ & $R 2 ; R 6$ & $R 3 ; R 5$ & $R 2$ & R3 & $R 4$ \\
\hline \multirow{4}{*}{ S1: Glycyl } & $\Delta G(k c a l / m o l)$ & -8.4 & -8.3 & -8.3 & -8.5 & -8.2 & -8.5 & -8.4 \\
\hline & $K\left(\times 10^{6}\right)$ & 1.44 & 1.21 & 1.21 & 1.70 & 1.02 & 1.70 & 1.44 \\
\hline & $K x / K C 60 p$ & 1.96 & 1.66 & 1.66 & 2.33 & 1.40 & 2.33 & 1.96 \\
\hline & Kx/Kxmin & 1.40 & 1.18 & 1.18 & 1.66 & 1.00 & 1.66 & 1.40 \\
\hline \multirow{4}{*}{$\begin{array}{l}\text { S2: } \\
\text { Cysteinyl }\end{array}$} & $\Delta G(k c a l / m o l)$ & -8.5 & -8.4 & -8.4 & -8.4 & -8.2 & -8.7 & -8.3 \\
\hline & $K\left(10^{6}\right)$ & 1.70 & 1.44 & 1.44 & 1.44 & 1.02 & 2.38 & 1.21 \\
\hline & $K x / K C 60 p$ & 2.33 & 1.96 & 1.96 & 1.96 & 1.40 & 3.26 & 1.66 \\
\hline & $K x / K x m i n$ & 1.66 & 1.40 & 1.40 & 1.40 & 1.00 & 2.33 & 1.18 \\
\hline \multirow{4}{*}{$\begin{array}{c}\text { S3: } \\
\text { Asparaginyl }\end{array}$} & $\Delta G(k c a l / m o l)$ & -8.8 & -8.6 & -8.6 & -8.8 & -8.7 & -8.9 & -8.6 \\
\hline & $K\left(\times 10^{6}\right)$ & 2.82 & 2.01 & 2.01 & 2.82 & 2.38 & 3.34 & 2.01 \\
\hline & $K x / K C 60 p$ & 3.86 & 2.75 & 2.75 & 3.86 & 3.26 & 4.57 & 2.75 \\
\hline & $K x / K x m i n$ & 1.40 & 1.00 & 1.00 & 1.40 & 1.18 & 1.66 & 1.00 \\
\hline \multirow{4}{*}{ S4: Lysyl } & $\Delta G(\mathrm{kcal} / \mathrm{mol})$ & -9.1 & -8.4 & -8.9 & -9.0 & -8.4 & -9.2 & -8.2 \\
\hline & $K\left(\times 10^{6}\right)$ & 4.68 & 1.44 & 3.34 & 3.95 & 1.44 & 5.54 & 1.02 \\
\hline & $K x / K C 60 p$ & 6.4 & 2.0 & 4.6 & 5.4 & 2.0 & 7.6 & 1.4 \\
\hline & $K x / K x m i n$ & 4.6 & 1.4 & 3.3 & 3.9 & 1.4 & 5.4 & 1.0 \\
\hline \multirow{4}{*}{ S5: Arginyl } & $\Delta G(\mathrm{kcal} / \mathrm{mol})$ & -9.5 & -8.9 & -9.2 & -9.4 & -8.7 & -8.3 & -8.4 \\
\hline & $K\left(\times 10^{6}\right)$ & 9.20 & 3.34 & 5.54 & 7.77 & 2.38 & 1.21 & 1.44 \\
\hline & $K x / K C 60 P$ & 12.6 & 4.6 & 7.6 & 10.6 & 3.3 & 1.7 & 2.0 \\
\hline & $K x / K x m i n$ & 7.6 & 2.8 & 4.6 & 6.4 & 2.0 & 1.0 & 1.2 \\
\hline \multirow{4}{*}{ S6: Glutam } & $\Delta G(k c a l / m o l)$ & -9.0 & -8.7 & -8.9 & -9.1 & -8.4 & -8.9 & -8.2 \\
\hline & $K\left(\times 10^{6}\right)$ & 3.95 & 2.38 & 3.34 & 4.68 & 1.44 & 3.34 & 1.02 \\
\hline & $K x / K C 60 p$ & 5.4 & 3.3 & 4.6 & 6.4 & 2.0 & 4.6 & 1.4 \\
\hline & $K x / K x m i n$ & 3.9 & 2.3 & 3.3 & 4.6 & 1.4 & 3.3 & 1.0 \\
\hline \multirow{4}{*}{ S7: Histydyl } & $\Delta G(k c a l / m o l)$ & -9.1 & -8.4 & -9.1 & -9.0 & -8.5 & -8.6 & -8.2 \\
\hline & $K\left(\times 10^{6}\right)$ & 4.68 & 1.44 & 4.68 & 3.95 & 1.70 & 2.01 & 1.02 \\
\hline & $K x / K C 60 p$ & 6.4 & 2.0 & 6.4 & 5.4 & 2.3 & 2.8 & 1.4 \\
\hline & $K x / K x m i n$ & 4.6 & 1.4 & 4.6 & 3.9 & 1.7 & 2.0 & 1.0 \\
\hline \multirow{4}{*}{$\begin{array}{c}\text { S8: } \\
\text { Phenylalanyl }\end{array}$} & $\Delta G(k c a l / m o l)$ & -9.1 & -8.4 & -9.1 & -9.8 & -8.5 & -8.6 & -8.2 \\
\hline & $K\left(\times 10^{6}\right)$ & 4.68 & 1.44 & 4.68 & 3.95 & 1.70 & 2.01 & 1.02 \\
\hline & Kx/KC60P & 12.6 & 4.6 & 7.6 & 20.9 & 6.4 & 4.6 & 1.4 \\
\hline & $K x / K x m i n$ & 9.0 & 3.3 & 5.4 & 14.9 & 4.6 & 3.3 & 1.0 \\
\hline \multirow{4}{*}{$\begin{array}{c}\text { S9: } \\
\text { Tryptophanyl }\end{array}$} & $\Delta G(k c a l / m o l)$ & -9.6 & -9.1 & -9.6 & -10.6 & -9.3 & -9.3 & -8.4 \\
\hline & $K\left(\times 10^{6}\right)$ & 10.89 & 4.68 & 10.89 & 58.87 & 6.56 & 6.56 & 1.44 \\
\hline & $K x / K C 60 p$ & 14.9 & 6.4 & 14.9 & 80.5 & 9.0 & 9.0 & 2.0 \\
\hline & $K x / K x m i n$ & 7.6 & 3.3 & 7.6 & 41.0 & 4.6 & 4.6 & 1.0 \\
\hline \multirow{4}{*}{ S10: Tyrosyl } & $\Delta G(k c a l / m o l)$ & -9.1 & -8.6 & -9.0 & -10.1 & -9.2 & -8.2 & -8.3 \\
\hline & $K\left(\times 10^{6}\right)$ & 4.68 & 2.01 & 3.95 & 25.32 & 5.54 & 1.02 & 1.21 \\
\hline & $K x / K C 60 p$ & 6.4 & 2.8 & 5.4 & 34.6 & 7.6 & 1.4 & 1.7 \\
\hline & Kx/Kxmin & 4.6 & 2.0 & 3.9 & 24.7 & 5.4 & 1.0 & 1.2 \\
\hline
\end{tabular}


Table 2. The values of binding affinity $\Delta \mathrm{G}(\mathrm{kcal} / \mathrm{mol})$ of the Lig2 molecule toward the proposed FF; $\mathrm{K}$ $\left(10^{6}\right)$ represents values of the binding constant; $K x / K C 60 p$ represent normalized factors describing an increase of the binding constant relative to the reference structure (C60_phen; $\Delta \mathrm{G}=-8.8(\mathrm{kcal} / \mathrm{mol})$; $\left.\mathrm{K}=2.82 \times 10^{6}\right) ; \mathrm{K} x / \mathrm{K} x \mathrm{~min}$ represent normalized factors describing an increase of the binding constant relative to the smallest values obtained for the functionalized fullerene with a particular substituent.

\begin{tabular}{|c|c|c|c|c|c|c|c|c|}
\hline \multirow{2}{*}{\multicolumn{2}{|c|}{$\begin{array}{c}\text { Quantity and Localization of } \\
\text { Substituents }\end{array}$}} & \multicolumn{7}{|c|}{ FF } \\
\hline & & $R 2 ; R 4 ; R 6$ & $R 2 ; R 4$ & $R 2 ; R 6$ & $R 3 ; R 5$ & $R 2$ & $R 3$ & $R 4$ \\
\hline \multirow{4}{*}{ S1: Glycyl } & $\Delta G(k c a l / m o l)$ & -9.2 & -9.3 & -9.1 & -9.2 & -9.2 & -9.1 & -9 \\
\hline & $K\left(\times 10^{6}\right)$ & 5.54 & 6.56 & 4.68 & 5.54 & 5.54 & 4.68 & 3.95 \\
\hline & $K x / K C 60 p$ & 1.96 & 2.33 & 1.66 & 1.96 & 1.96 & 1.66 & 1.40 \\
\hline & Kx/Kxmin & 1.40 & 1.66 & 1.18 & 1.40 & 1.40 & 1.18 & 1.00 \\
\hline \multirow{4}{*}{$\begin{array}{c}\text { S2: } \\
\text { Cysteinyl }\end{array}$} & $\Delta G(k c a l / m o l)$ & -9.3 & -9.2 & -9.1 & -9.3 & -9 & -9.1 & -9.1 \\
\hline & $K\left(10^{6}\right)$ & 6.56 & 5.54 & 4.68 & 6.56 & 3.95 & 4.68 & 4.68 \\
\hline & $K x / K C 60 p$ & 2.33 & 1.96 & 1.66 & 2.33 & 1.40 & 1.66 & 1.66 \\
\hline & Kx/Kxmin & 1.66 & 1.40 & 1.18 & 1.66 & 1.00 & 1.18 & 1.18 \\
\hline \multirow{4}{*}{$\begin{array}{c}\text { S3: } \\
\text { Asparaginyl }\end{array}$} & $\Delta G(k c a l / m o l)$ & -9.5 & -9.5 & -9.4 & -9.6 & -9.4 & -9.2 & -9.4 \\
\hline & $K\left(\times 10^{6}\right)$ & 9.20 & 9.20 & 7.77 & 10.89 & 7.77 & 5.54 & 7.77 \\
\hline & $K x / K C 60 p$ & 3.26 & 3.26 & 2.75 & 3.86 & 2.75 & 1.96 & 2.75 \\
\hline & Kx/Kxmin & 1.66 & 1.66 & 1.40 & 1.96 & 1.40 & 1.00 & 1.40 \\
\hline \multirow{4}{*}{ S4: Lysyl } & $\Delta G(k c a l / m o l)$ & -9.6 & -9.6 & -9.2 & -9.9 & -9.5 & -9.2 & -8.9 \\
\hline & $K\left(\times 10^{6}\right)$ & 10.89 & 10.89 & 5.54 & 18.06 & 9.20 & 5.54 & 3.34 \\
\hline & $K x / K C 60 p$ & 3.9 & 3.9 & 2.0 & 6.4 & 3.3 & 2.0 & 1.2 \\
\hline & Kx/Kxmin & 3.3 & 3.3 & 1.7 & 5.4 & 2.8 & 1.7 & 1.0 \\
\hline \multirow{4}{*}{ S5: Arginyl } & $\Delta G(k c a l / m o l)$ & -9.8 & -9.6 & -9.5 & -10.1 & -9.1 & -9.5 & -9.0 \\
\hline & $K\left(\times 10^{6}\right)$ & 15.26 & 10.89 & 9.20 & 25.32 & 4.68 & 9.20 & 3.95 \\
\hline & Кх/КС60Р & 5.4 & 3.9 & 3.3 & 9.0 & 1.7 & 3.3 & 1.4 \\
\hline & Kx/Kxmin & 3.9 & 2.8 & 2.3 & 6.4 & 1.2 & 2.3 & 1.0 \\
\hline \multirow{4}{*}{ S6: Glutam } & $\Delta G(\mathrm{kcal} / \mathrm{mol})$ & -9.6 & -9.4 & -9.4 & -9.7 & -9.1 & -9.4 & -8.9 \\
\hline & $K\left(\times 10^{6}\right)$ & 10.89 & 7.77 & 7.77 & 12.89 & 4.68 & 7.77 & 3.34 \\
\hline & $K x / K C 60 p$ & 3.9 & 2.8 & 2.8 & 4.6 & 1.7 & 2.8 & 1.2 \\
\hline & Kx/Kxmin & 3.3 & 2.3 & 2.3 & 3.9 & 1.4 & 2.3 & 1.0 \\
\hline \multirow{4}{*}{ S7: Histydyl } & $\Delta G(k c a l / m o l)$ & -9.6 & -9.8 & -9.9 & -10.0 & -9.2 & -9.2 & -8.9 \\
\hline & $K\left(\times 10^{6}\right)$ & 10.89 & 15.26 & 18.06 & 21.38 & 5.54 & 5.54 & 3.34 \\
\hline & $K x / K C 60 p$ & 3.9 & 5.4 & 6.4 & 7.6 & 2.0 & 2.0 & 1.2 \\
\hline & Kx/Kxmin & 3.3 & 4.6 & 5.4 & 6.4 & 1.7 & 1.7 & 1.0 \\
\hline \multirow{4}{*}{$\begin{array}{c}\text { S8: } \\
\text { Phenylalanyl }\end{array}$} & $\Delta G(\mathrm{kcal} / \mathrm{mol})$ & -9.9 & -9.5 & -9.9 & -10.8 & -9.7 & -9.4 & -8.9 \\
\hline & $K\left(\times 10^{6}\right)$ & 18.06 & 9.20 & 18.06 & 82.51 & 12.89 & 7.77 & 3.34 \\
\hline & Кх /КС60Р & 6.4 & 3.3 & 6.4 & 29.2 & 4.6 & 2.8 & 1.2 \\
\hline & Kx/Kxmin & 5.4 & 2.8 & 5.4 & 24.7 & 3.9 & 2.3 & 1.0 \\
\hline \multirow{4}{*}{$\begin{array}{c}\text { S9: } \\
\text { Tryptophanyl }\end{array}$} & $\Delta G(k c a l / m o l)$ & -9.7 & -9.7 & -9.6 & -11.0 & -9.5 & -9.3 & -9.0 \\
\hline & $K\left(\times 10^{6}\right)$ & 12.89 & 12.89 & 10.89 & 115.64 & 9.20 & 6.56 & 3.95 \\
\hline & $K x / K C 60 p$ & 4.6 & 4.6 & 3.9 & 41.0 & 3.3 & 2.3 & 1.4 \\
\hline & Kx/Kxmin & 3.3 & 3.3 & 2.8 & 29.2 & 2.3 & 1.7 & 1.0 \\
\hline \multirow{4}{*}{ S10: Tyrosyl } & $\Delta G(\mathrm{kcal} / \mathrm{mol})$ & -9.7 & -9.6 & -9.7 & -10.8 & -9.1 & -8.9 & -9.0 \\
\hline & $K\left(\times 10^{6}\right)$ & 12.89 & 10.89 & 12.89 & 82.51 & 4.68 & 3.34 & 3.95 \\
\hline & $K x / K C 60 p$ & 4.6 & 3.9 & 4.6 & 29.2 & 1.7 & 1.2 & 1.4 \\
\hline & Kx/Kxmin & 3.9 & 3.3 & 3.9 & 24.7 & 1.4 & 1.0 & 1.2 \\
\hline
\end{tabular}

The increasing of molecule length by one or two additional carbon atoms in the side chain of the amino acids relative to the previously discussed group of substituents significantly increased the binding capabilities of the created nanomolecules. In this case, there is a higher variety of obtained The highest values are noticed for nanomolecules with residues in positions R3 and R5. The functionalized fullerenes with three substituents (R2; R4; R6) exhibit the highest binding affinities toward the Lig1 molecule $(\mathrm{Kx} / \mathrm{Kxmin}=7.6$, S5: Arginyl); however, the difference compared to the best two substituted systems is quite small.

The values presented in Table 2, characterizing interactions of the Lig2 molecule with disubstituted nanomolecules, clearly show that FF with residues in positions R2; R4 and R2; R6 exhibit very similar 
binding properties, slightly higher than appropriate mono derivatives. The substitution in positions R3; R5 obtains nanomolecules exhibiting the best binding properties $(\mathrm{Kx} / \mathrm{Kxmin}=6.4$, S5: Arginyl), even better than in the case of the three substituted derivatives (R2; R4; R6). The last group of substituents is amino acids that contain cyclic systems localized in the side chain of molecules, namely histidine, phenylalanine, tryptophan and tyrosine. The use of substituents with aromatic systems efficiently increases the binding capabilities of created FF, which was also found in previous studies using immobilized fullerenes [14,32]. In this case, analogically, like for previous groups of substituents, the smallest values of binding affinity were reported for monosubstituted derivatives with residues placed in position R4, while for the remaining two types of substitution (R2 and R3), there can be found conformations that exhibit binding properties similar to disubstituted nanomolecules. The data presented in Tables 1 and 2 clearly show that the localization of substituents in the case of derivatives containing aromatic rings with two residues significantly affects the binding capabilities of ligands by such structures. It can be stated that substitution in positions R3; R5 is more favorable than in the case of both remaining types, which is unambiguously confirmed in the differences in values of the binding affinities and appropriate constants. The appearance of the third substituent (R2; R3; R6) in the aromatic ring does not improve the binding capabilities of such nanomolecules, which are similar to substitution systems R2; R4 and R2; R6. Among all 70 nanostructures obtained by the use of the proposed substituents, one may choose systems exhibiting the highest binding capabilities relative to both considered ligands. Appropriate complexes of selected nanocarriers are presented in Figures 3 and 4 . The native nanomolecule $\mathrm{C} 60$-phen creates complexes with ligands that are maintained mainly by stacking interactions between the aromatic rings of ligands and the surface of the fullerene [27,28]. The graphic representation of such a complex is presented in Figure 3a. The observed distances range from 3.27 to $3.73 \AA$ and a planar orientation between interacting cyclic systems confirms the dominant role of such interactions. In the case of the Lig1 molecule, three nanomolecules that exhibit significant binding activity in the creation of complexes deserve special attention. All of them are FF derivatives with substituents placed in positions R3; R5. The first of them is FF_R3_R5_Phenylalanyl, which exhibits $20.9(\mathrm{Kx} / \mathrm{KC} 60 \mathrm{p})$ times higher binding capabilities than the reference system. Figure $3 \mathrm{~b}$ shows that conformation of the ligand molecule ensures the occurrence of stacking interactions with the fullerene surface; however, additional interactions are also present, namely hydrogen bonds, occurring between carbonyl oxygen atoms of the nanomolecule and hydrogens from amide ( $2.46 \AA$ ) and amine $(2.36 \AA)$ groups of the ligand molecule. The next stabilizing factor is the creation of a characteristic cluster of stacking molecules which consists of the surface of the fullerene, the oxindole ligand core and a ring from a phenylalanine substituent, which is confirmed by mutual distances ( $3.49 \AA, 3.69 \AA$ ) and orientations of the mentioned subunits. The next interesting molecule is FF_R3_R5_Tyrosyl for which a 34.6-time increase of complex binding compared to the reference molecule was noticed. Figure $3 \mathrm{c}$ clearly shows that the complex created by this nanomolecule is maintained by a network of stabilizing interactions. The stacking interactions are observed between all cyclic elements of the ligand molecule and the fullerene surface (distance from 3.39 to $3.59 \AA$ ). Analogically, like in the case of the previously discussed complex, the creation of a cluster of stacking molecules is observed, created by the aromatic ring of tyrosine, the ligand core and the fullerene surface. The second factor stabilizing the ligand complex with the considered FF are three hydrogen bonds. The first of them is localized between the carbonyl oxygen atom and hydrogen from the amide group ( $2.27 \AA)$; the next two are created by hydrogen bond donors and acceptors from the five-membered ring of the ligand and hydroxyl group of tyrosine (2.20 and $2.31 \AA$ ). Among all considered nanomolecules, the highest binding affinity $(-10.6 \mathrm{kcal} / \mathrm{mol})$ was noticed for FF_R3_R5_Tryptophanyl. In this case, an 80.5 (Kx/KC60p) times increase of binding capabilities was found relative to the native structure. The graphic representation of the complex of this nanocarrier with Lig1 molecules is presented in Figure 3d. The presented structure is stabilized by numerous stacking interactions in which all cyclic systems of the ligand molecule (distances from 3.50 to $3.69 \AA$ ) are involved. 


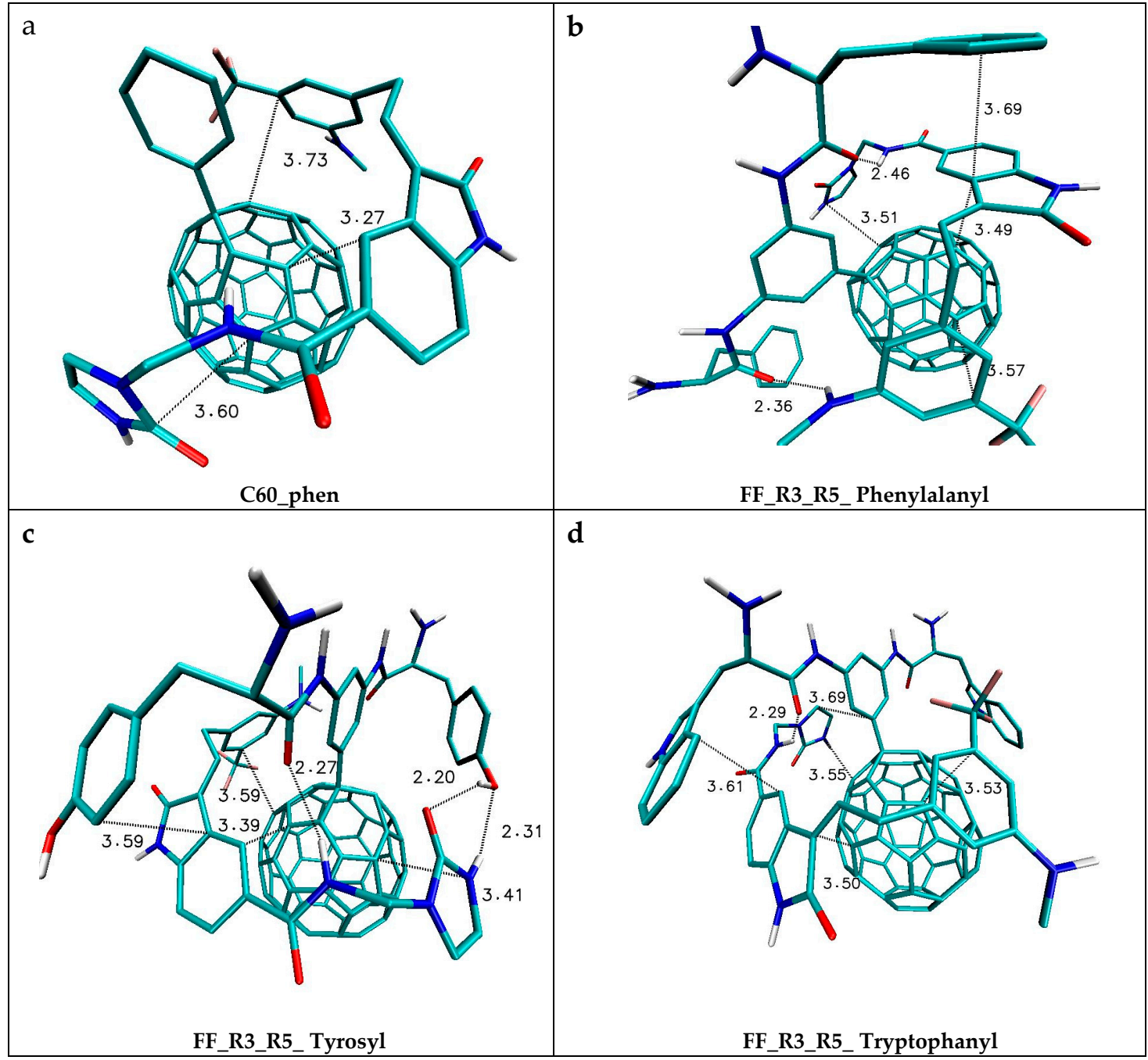

Figure 3. The graphic representation of chosen complexes created by the Lig1 molecule with FF exhibiting the best binding capabilities. The atoms of particular chemical elements are indicated by following colors: carbon: cyan; nitrogen: blue; oxygen: red; hydrogen: white; fluorine: pink.

There is also observed a cluster of stacking systems created by cyclic systems of tryptophan, ligand core and fullerene. The orientation of the five-membered ring of the ligand indicates the possibility of interaction with fullerene surface ( $3.55 \AA$ ) and also with benzene ring ( $3.69 \AA)$ substituted to the surface of the nanomolecule.

The analyzed ligand molecule, namely indol_4_9, was a subject of analogical investigations realized with the use of functionalized fullerenes. The nanomolecules used in this case were also C60 fullerene derivatives that contained different active groups in their structure including aliphatic, halogen and aromatic substituents attached to the fullerene surface. Among all obtained complexes, the best affinity was found for the nanocarrier functionalized by three additional aromatic systems; however, the obtained value of affinity was equal to $-8.7(\mathrm{kcal} / \mathrm{mol})$ [32]. The outcomes obtained for the FF used in this work suggest that they have higher binding capabilities than previously considered nanomolecules. In the case of the best nanocarrier, a 24.7 times increase of the binding constant compared to the previously considered fullerenes is observed. The proposed procedure of the C60 fullerene molecule functionalization allowed for obtaining nanocarriers able to utilize the binding capabilities of the considered ligand molecule better.

The graphic characteristics of the chosen complexes created by the Lig2 molecule are presented in Figure 4 . Among all tested systems, the complexes characterized by the highest values of the binding 
affinity deserve special attention. The FF_R3_R5_Arginyl molecule (Figure 4a) creates complexes characterized by a binding affinity value equal to $-10.1 \mathrm{kcal} / \mathrm{mol}$, giving a $9.0(\mathrm{Kx} / \mathrm{KC} 60 \mathrm{p})$ times higher increase of binding capabilities relative to the native molecule. The considered complex is maintained by two types of interactions. All cyclic systems of the Lig2 molecule are involved in the creation of stacking interactions with the fullerene surface (dist. from 3.36 to $3.56 \AA$ ).

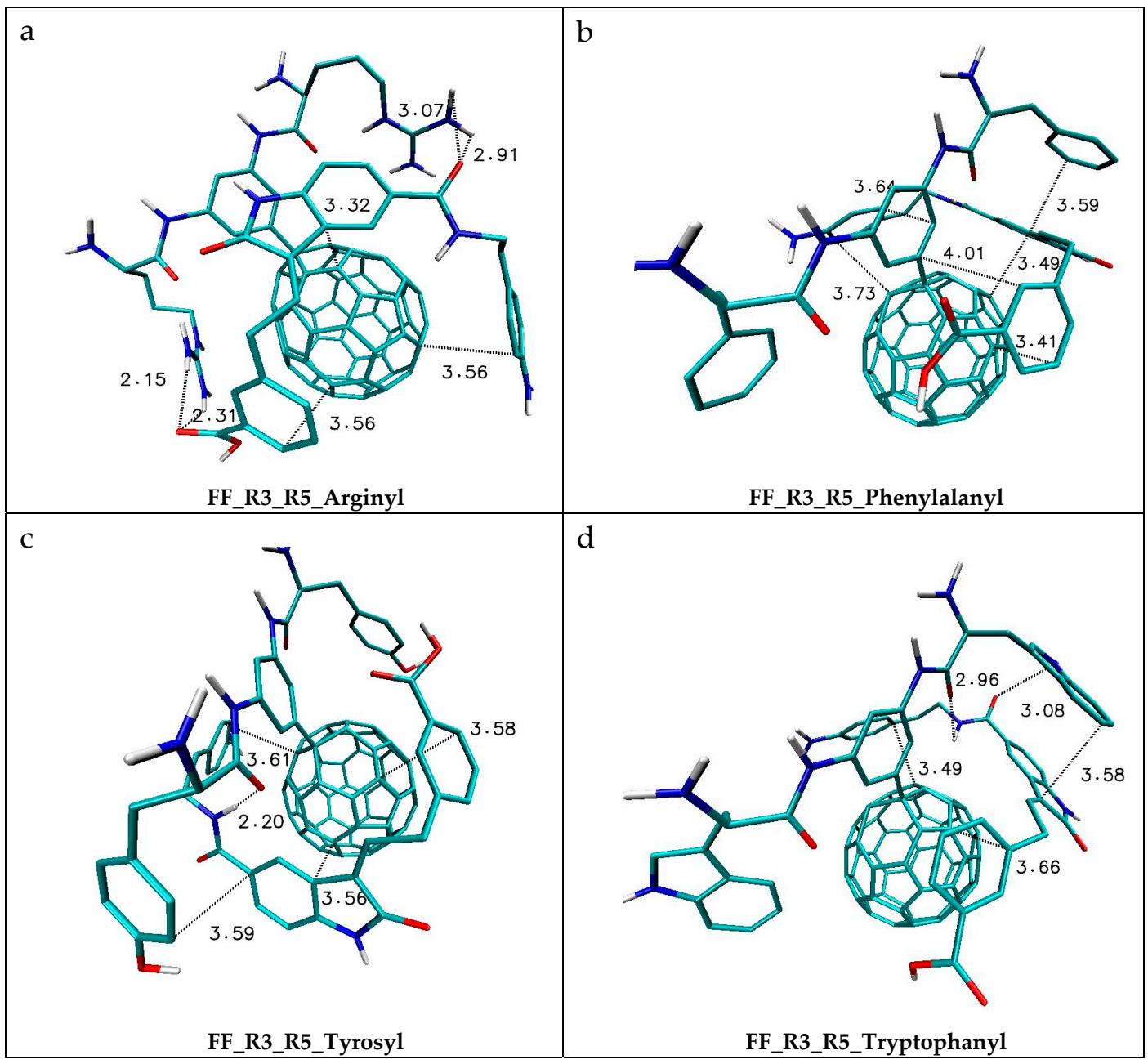

Figure 4. The graphic representation of the chosen complexes created by the Lig2 molecule with FF exhibiting the best binding capabilities. The atoms of particular chemical elements are indicated by the following colors: carbon: cyan; nitrogen: blue; oxygen: red; hydrogen: white.

The orientation of the ligand relative to the fullerene molecule also provides the occurrence of hydrogen bonds created by hydrogens from terminal amino groups of both arginine substituents (lengths from 2.15 to $2.91 \AA$ ). The next two nanostructures, namely FF_R3_R5_Phenylalanyl (Figure 4b) and FF_R3_R5_Tyrosyl (Figure 4c), exhibit similar binding affinity toward the ligand molecule $(-10.8 \mathrm{kcal} / \mathrm{mol})$, which makes the binding capability of such molecules 29.2 times higher than in the case of the reference molecule. Both complexes are maintained mainly by stacking interactions occurring between all cyclic systems of the ligand and surface of the fullerene, and additional stacking contribution is related to the presence of stacking clusters created by aromatic systems of amino acids, the core of the ligand molecule and the fullerene surface. One hydrogen bond was found in the structure of the FF_R3_R5_Tyrosyl complex, created by a carbonyl oxygen atom from the fullerene and amide hydrogen from the ligand molecule $(2.20 \AA)$. The highest activity in the creation of complexes with the Lig2 molecule among all considered nanomolecules is exhibited by FF_R3_R5_Tryptophanyl $(-11.0 \mathrm{kcal} / \mathrm{mol})$, which is associated with a 41 times increase in the binding capacity by this nanoparticle 
compared to the reference molecule. The structure of complexes of this FF is mainly maintained by stacking interactions in which all cyclic systems of the ligand molecule are involved, which is confirmed by mutual distances measured between aromatic systems and their mutual orientation. Similarly, as in the case of the previous FF, the aromatic system from the tryptophanyl substituent is involved in the creation of a stacking cluster with the central core of the Lig2 molecule. The structural analysis of the considered complexes indicates also the possibility of the occurrence of hydrogen bonds; however, the observed interactions are weak and their role in complex stabilization is less significant $[13,14]$. The Lig 2 molecule (indol_20_10), analogically like the Lig 1 molecule, was used in previous studies related to immobilization with the use of $\mathrm{C} 60$ derivatives. This particular molecule exhibited good binding affinity toward the considered nanosystems, better than the second considered drug which was confirmed by the highest value of binding affinity equal to $-9.2(\mathrm{kcal} / \mathrm{mol})$ [32]. The comparison between this value and the results recorded for the FF containing amino acid substituents unambiguously confirms the superiority of the new class of nanostructures (FF_R3_R5_Tryptophanyl; $-11.0 \mathrm{kcal} / \mathrm{mol}$, 20.9 times K-bind increase). The most important factor in differentiating between the compared classes of nanosystems is related to the localization of active groups. The addition of substituents to the aromatic system on the fullerene surface significantly increases the conformational capabilities of amino acids, which significantly affects the ligand-binding capacity. The most important effects of increased conformational abilities of substituents are observed for aromatic amino acids, which participate in the creation of stacking clusters with the ligand molecule core and the fullerene surface.

\section{Conclusions}

The immobilization of drugs with the use of nanocarriers is one of the strategies during the creation of targeted therapies. One of the most important aspects of such methods is the appropriate matching of nanoparticles to transferred drugs. The set of nanomolecules analyzed in this work exhibits significant diversity allowing for definition of the optimal type and localization of substituents ensuring best-fitting analyzed drug molecules. The obtained data show that substituents with a short length (glycine, cysteine or asparagine), even though they contain hydrogen bond donors or acceptors, are not able to significantly increase the binding capability of the considered ligand molecules. In this case, the quantity and localization of the used chemical groups in the aromatic systems do not significantly affect the considered properties. The use of amino acids with active groups localized on longer side chains (lysine, arginine and glutamine) had a larger impact on the binding properties of the obtained nanomolecules. A larger conformational flexibility of such substituents caused their quantity and place of substitution to induce noticeable changes in the properties of the obtained FF. In the case of the monosubstitued derivatives, there is a noticeable increase in the binding capabilities in the order $\mathrm{R} 4<\mathrm{R} 2<\mathrm{R} 3$. The nanostructures with two substituents mainly exhibit higher binding affinities than the systems with one residue. Among all the disubstituted systems, the best effects are observed in the case of substitution realized in positions R3 and R5. Among all the used substituents, the best effects were obtained in the case of the amino acids containing aromatic cyclic systems in their structure. The FF obtained by addition of tryptophanyl, phenylalanyl or tyrosyl residues in positions R3; R5 exhibit the best binding capabilities relative to both analyzed ligands. Such a location of the considered amino acids in the phenyl ring ensures the possibility of the creation of characteristic stacking clusters consisting of three stacking aromatic subsystems in which the main core of the ligand molecule is placed between the fullerene surface and aromatic systems of the amino acids. The presence of this additional stabilizing impact can explain significantly higher binding capabilities of such nanomolecules relative to the ligand molecules. The analysis of the binding capabilities of all considered nanocarriers, incorporating also the quantity and type of interactions involved in the stabilization of complexes, facilitates the conclusion that, in such systems, hydrophobic and stacking interactions are preferred while hydrogen bonds perform a supporting function.

The obtained results clearly indicate a group of substituents and substitution sites that provide the most stable complexes that can be used to create new nanocarriers for considered drugs. Selected 
nanocompounds are a solid base for further research carried out based on more advanced computational methods such as molecular dynamics that determine the essence of interactions responsible for the stabilization of created complexes. A more in-depth understanding of the proposed complexes is crucial in the context of the selection of systems for potential synthesis and their use in the creation of targeted therapy.

Author Contributions: Conceptualization, P.C.; Methodology, P.C.; Software, P.C.; Validation, P.C.; Formal Analysis, P.C.; Investigation, P.C. and B.S.; Resources, P.C. and B.S.; Data Curation, P.; Writing-Original Draft Preparation, P.C.; Writing—Review \& Editing, P.C. and B.S.; Visualization, P.C.; Supervision, P.C.; Project Administration, P.C. All authors have read and agree to the published version of the manuscript.

Funding: This research received no external funding.

Acknowledgments: This research was supported by PL-Grid Infrastructure (http://www.plgrid.pl/en).

Conflicts of Interest: The authors declare that there are no conflicts of interest.

\section{References}

1. Damascelli, B.; Patelli, G.L.; Lanocita, R.; Tolla, G.D.; Frigerio, L.F.; Marchianò, A.; Garbagnati, F.; Spreafico, C.; Tichà, V.; Gladin, C.R.; et al. A Novel Intraarterial Chemotherapy Using Paclitaxel in Albumin Nanoparticles to Treat Advanced Squamous Cell Carcinoma of the Tongue: Preliminary Findings. Am. J. Roentgenol. 2003, 181, 253-260. [CrossRef]

2. Dyer, A.M.; Hinchcliffe, M.; Watts, P.; Castile, J.; Jabbal-Gill, I.; Nankervis, R.; Smith, A.; Illum, L. Nasal delivery of insulin using novel chitosan based formulations: A comparative study in two animal models between simple chitosan formulations and chitosan nanoparticles. Pharm. Res. 2002, 19, 998-1008. [CrossRef]

3. Huang, M.; Khor, E.; Lim, L.-Y. Uptake and cytotoxicity of chitosan molecules and nanoparticles: Effects of molecular weight and degree of deacetylation. Pharm. Res. 2004, 21, 344-353. [CrossRef]

4. Cascone, M.G.; Lazzeri, L.; Carmignani, C.; Zhu, Z. Gelatin nanoparticles produced by a simple W/O emulsion as delivery system for methotrexate. J. Mater. Sci. Mater. Med. 2002, 13, 523-526. [CrossRef]

5. Hainfeld, J.F.; Slatkin, D.N.; Smilowitz, H.M. The use of gold nanoparticles to enhance radiotherapy in mice. Phys. Med. Biol. 2004, 49, N309-N315. [CrossRef]

6. Paciotti, G.F.; Myer, L.; Weinreich, D.; Goia, D.; Pavel, N.; McLaughlin, R.E.; Tamarkin, L. Colloidal Gold: A Novel Nanoparticle Vector for Tumor Directed Drug Delivery. Drug Deliv. 2004, 11, 169-183. [CrossRef]

7. Gupta, M.; Gupta, A.K. Hydrogel pullulan nanoparticles encapsulating pBUDLacZ plasmid as an efficient gene delivery carrier. J. Control. Release 2004, 99, 157-166. [CrossRef]

8. Gupta, A.K.; Gupta, M. Synthesis and surface engineering of iron oxide nanoparticles for biomedical applications. Biomaterials 2005, 26, 3995-4021. [CrossRef]

9. Alyautdin, R.N.; Petrov, V.E.; Langer, K.; Berthold, A.; Kharkevich, D.A.; Kreuter, J. Delivery of loperamide across the blood-brain barrier with polysorbate 80-coated polybutylcyanoacrylate nanoparticles. Pharm. Res. 1997, 14, 325-328. [CrossRef]

10. Kreuter, J.; Ramge, P.; Petrov, V.; Hamm, S.; Gelperina, S.E.; Engelhardt, B.; Alyautdin, R.; von Briesen, H.; Begley, D.J. Direct evidence that polysorbate-80-coated poly(butylcyanoacrylate) nanoparticles deliver drugs to the CNS via specific mechanisms requiring prior binding of drug to the nanoparticles. Pharm. Res. 2003, 20, 409-416. [CrossRef]

11. Weissenböck, A.; Wirth, M.; Gabor, F. WGA-grafted PLGA-nanospheres: Preparation and association with Caco-2 single cells. J. Control. Release 2004, 99, 383-392. [CrossRef]

12. Czeleń, P.; Szefler, B. The Immobilization of Oxindole Derivatives with Use of Cube Rhombellane Homeomorphs. Symmetry 2019, 11, 900. [CrossRef]

13. Szefler, B.; Czeleń, P. Docking of Cisplatin on Fullerene Derivatives and Some Cube Rhombellane Functionalized Homeomorphs. Symmetry 2019, 11, 874. [CrossRef]

14. Czeleń, P.; Szefler, B. The Immobilization of ChEMBL474807 Molecules Using Different Classes of Nanostructures. Symmetry 2019, 11, 980. [CrossRef]

15. Szefler, B. Nanotechnology, from quantum mechanical calculations up to drug delivery. Int. J. Nanomed. 2018, 13, 6143-6176. [CrossRef] [PubMed] 
16. De Jong, W.H.; Borm, P.J.A. Drug delivery and nanoparticles:applications and hazards. Int. J. Nanomed. 2008, 3, 133-149. [CrossRef]

17. Cataldo, F.; Da Ros, T. Medicinal Chemistry and Pharmacological Potential of Fullerenes and Carbon Nanotubes; Springer: Dordrecht, The Netherlands, 2008; ISBN 9781402068454.

18. Panchuk, R.R.; Prylutska, S.V.; Chumakl, V.V.; Skorokhyd, N.R.; Lehka, L.V.; Evstigneev, M.P.; Prylutskyy, Y.I.; Berger, W.; Heffeter, P.; Scharff, P.; et al. Application of $\mathrm{C}_{60}$ Fullerene-Doxorubicin Complex for Tumor Cell Treatment In Vitro and In Vivo. J. Biomed. Nanotechnol. 2015, 11, 1139-1152. [CrossRef]

19. Gao, Z.; Zhang, L.; Sun, Y. Nanotechnology applied to overcome tumor drug resistance. J. Control. Release 2012, 162, 45-55. [CrossRef]

20. Turov, V.V.; Chehun, V.F.; Barvinchenko, V.N.; Krupskaya, T.V.; Prylutskyy, Y.I.; Scharff, P.; Ritter, U. Low-temperature 1H-NMR spectroscopic study of doxorubicin influence on the hydrated properties of nanosilica modified by DNA. J. Mater. Sci. Mater. Med. 2011, 22, 525-532. [CrossRef]

21. Morgen, M.; Bloom, C.; Beyerinck, R.; Bello, A.; Song, W.; Wilkinson, K.; Steenwyk, R.; Shamblin, S. Polymeric Nanoparticles for Increased Oral Bioavailability and Rapid Absorption Using Celecoxib as a Model of a Low-Solubility, High-Permeability Drug. Pharm. Res. 2012, 29, 427-440. [CrossRef]

22. Andrievsky, G.; Klochkov, V.; Derevyanchenko, L. Is the $\mathrm{C}_{60}$ Fullerene Molecule Toxic?! Fuller. Nanotub. Carbon Nanostructures 2005, 13, 363-376. [CrossRef]

23. D'Rozario, R.S.G.; Wee, C.L.; Jayne Wallace, E.; Sansom, M.S.P. The interaction of $C_{60}$ and its derivatives with a lipid bilayer via molecular dynamics simulations. Nanotechnology 2009, 20, 115102. [CrossRef] [PubMed]

24. Qiao, R.; Roberts, A.P.; Mount, A.S.; Klaine, S.J.; Ke, P.C. Translocation of $\mathrm{C}_{60}$ and Its Derivatives Across a Lipid Bilayer. Nano Lett. 2007, 7, 614-619. [CrossRef] [PubMed]

25. Prylutska, S.; Bilyy, R.; Overchuk, M.; Bychko, A.; Andreichenko, K.; Stoika, R.; Rybalchenko, V.; Prylutskyy, Y.; Tsierkezos, N.G.; Ritter, U. Water-soluble pristine fullerenes $\mathrm{C}_{60}$ increase the specific conductivity and capacity of lipid model membrane and form the channels in cellular plasma membrane. J. Biomed. Nanotechnol. 2012, 8, 522-527. [CrossRef]

26. Schuetze, C.; Ritter, U.; Scharff, P.; Fernekorn, U.; Prylutska, S.; Bychko, A.; Rybalchenko, V.; Prylutskyy, Y. Interaction of $\mathrm{N}$-fluorescein-5-isothiocyanate pyrrolidine- $\mathrm{C}_{60}$ with a bimolecular lipid model membrane. Mater. Sci. Eng. C 2011, 31, 1148-1150. [CrossRef]

27. Yang, X.; Ebrahimi, A.; Li, J.; Cui, Q. Fullerene-biomolecule conjugates and their biomedicinal applications. Int. J. Nanomed. 2014, 9, 77-92. [CrossRef]

28. Montellano, A.; Da Ros, T.; Bianco, A.; Prato, M. Fullerene $\mathrm{C}_{60}$ as a multifunctional system for drug and gene delivery. Nanoscale 2011, 3, 4035. [CrossRef]

29. Luk, K.-C.; Simcox, M.E.; Schutt, A.; Rowan, K.; Thompson, T.; Chen, Y.; Kammlott, U.; DePinto, W.; Dunten, P.; Dermatakis, A. A new series of potent oxindole inhibitors of CDK2. Bioorg. Med. Chem. Lett. 2004, 14, 913-917. [CrossRef]

30. Bramson, H.N.; Corona, J.; Davis, S.T.; Dickerson, S.H.; Edelstein, M.; Frye, S.V.; Gampe, R.T.; Harris, P.A.; Hassell, A.; Holmes, W.D.; et al. Oxindole-Based Inhibitors of Cyclin-Dependent Kinase 2 (CDK2): Design, Synthesis, Enzymatic Activities, and X-ray Crystallographic Analysis. J. Med. Chem. 2001, 44, 4339-4358. [CrossRef]

31. Dermatakis, A.; Luk, K.C.; DePinto, W. Synthesis of potent oxindole CDK2 inhibitors. Bioorg. Med. Chem. 2003, 11, 1873-1881. [CrossRef]

32. Czelen, P. Investigation of the Inhibition Potential of New Oxindole Derivatives and Assessment of Their Usefulness for Targeted Therapy. Symmetry 2019, 11, 974. [CrossRef]

33. Kornev, A.B.; Khakina, E.A.; Troyanov, S.I.; Kushch, A.A.; Peregudov, A.; Vasilchenko, A.; Deryabin, D.G.; Martynenko, V.M.; Troshin, P.A. Facile preparation of amine and amino acid adducts of [60]fullerene using chlorofullerene $\mathrm{C}_{60} \mathrm{C}_{16}$ as a precursor. Chem. Commun. 2012, 48, 5461. [CrossRef] [PubMed]

34. Kim, K.-H.; Ko, D.K.; Kim, Y.-T.; Kim, N.H.; Paul, J.; Zhang, S.-Q.; Murray, C.B.; Acharya, R.; Kim, Y.H.; DeGrado, W.F.; et al. Protein-directed self-assembly of a fullerene crystal. Nat. Commun. 2016, 7, 11429. [CrossRef] [PubMed]

35. PubChem. Available online: https://pubchem.ncbi.nlm.nih.gov/ (accessed on 1 May 2019).

36. Trott, O.; Olson, A.J. AutoDock Vina: Improving the speed and accuracy of docking with a new scoring function, efficient optimization, and multithreading. J. Comput. Chem. 2010, 31, 455-461. [CrossRef] 
37. Bartashevich, E.V.; Potemkin, V.A.; Grishina, M.A.; Belik, A.V. A Method for Multiconformational Modeling of the Three-Dimensional Shape of a Molecule. J. Struct. Chem. 2002, 43, 1033-1039. [CrossRef]

38. Humphrey, W.; Dalke, A.; Schulten, K. VMD: Visual molecular dynamics. J. Mol. Graph. 1996, 14, $33-38$. [CrossRef]

39. Castro-Alvarez, A.; Costa, A.M.; Vilarrasa, J. The Performance of several docking programs at reproducing protein-macrolide-like crystal structures. Molecules 2017, 22, 136. [CrossRef] 